\title{
Are patients with poor left ventricular function more prone to oxidative stress during cardiac surgery?
}

In this issue, De Vecchi and colleagues report an association between preoperative ventricular function and the degree of myocardial oxidative stress occurring during coronary artery bypass surgery. ${ }^{1}$ They found a relation between left ventricular ejection fraction and myocardial glutathione concentrations and suggest that hearts with the poorest function have the weakest defences against oxidative stresses induced by ischaemia and reperfusion. The study has explored the subject from a novel viewpoint and if the findings are substantiated they have implications for myocardial protection in clinical practice.

Since the early 1980s it has been accepted that oxygen derived free radicals are involved in myocardial injury following ischaemia and reperfusion; therefore, free radicals have been of great interest to cardiac surgeons. ${ }^{2}$ A free radical is simply a molecule with an unpaired electron that makes it highly reactive and potentially damaging to lipid cell membranes and proteins. Free radicals are produced during normal physiological conditions but are inactivated by intracellular enzymatic scavengers - for example, superoxide dismutase and catalase, or by thiol containing antioxidants reduced glutathione and vitamin $\mathrm{E}$. When these defences are exhausted by ischaemia and by the burst of free radicals produced during reperfusion, myocardial injury occurs. This damage, often termed reperfusion injury, is manifest mainly by arrhythmias and a temporary depression in myocardial contractility-stunning. ${ }^{34}$ The current understanding of the pathophysiology of stunning implicates oxygen derived free radicals in contractile protein damage that leads to impaired responsiveness to the oscillations in intracellular calcium concentrations and hence reduced contractility. Free radicals are extremely difficult to measure as the kinetics of their reactions are so short, and therefore in many experiments the levels of antioxidant defences or products of oxidative damage are assayed as an indirect measure of the "oxidant stress".

Most of the studies published to date are animal experiments in which agents thought to reduce free radicals have been administered in an attempt to attenuate myocardial injury following ischaemia and reperfusion. There is also evidence for free radical injury occurring in people during cardiac surgery using cardiopulmonary bypass. ${ }^{5}$ Ferrari and colleagues were the first to demonstrate myocardial oxidative stress in man by measuring the release of oxidised glutathione in the coronary sinus following reperfusion during cardiac surgery. ${ }^{6}$ They showed a positive correlation between the ischaemic time (when above 30 minutes) and the amount of glutathione release. The patients with longer ischaemic times and more oxidative damage had lower cardiac indexes in the first six hours after surgery. Since then surgeons have attempted to replicate the animal experiments by increasing oxidative defences to reduce ischaemia-reperfusion injury. This has not always been as successful in clinical practice as in the laboratory. ${ }^{7}$

This summary explains how oxidative stress might temporarily reduce ventricular function; however, until now no one had demonstrated that hearts with impaired ventricu- lar function were more vulnerable to oxidative stress. This subject is complex and can be difficult to investigate in controlled laboratory conditions and we must therefore congratulate De Vecchi and colleagues for tackling the problem in patients having cardiac surgery.

However, the study reported by De Vecchi is not perfect. The most important finding, that total myocardial glutathione in left ventricular biopsy specimens correlated with left ventricular ejection fraction at the beginning of bypass (before aortic cross clamping), must be examined more closely. First there may be sampling errors because of regional variations in the myocardium that could account for some results. Many patients in the study had suffered previous myocardial infarctions and would therefore be expected to have scar tissue that could have contaminated the biopsies. Second, is the overreliance on correlation. The finding of associations between variables is relatively common in biological data and a significant correlation coefficient does not necessarily indicate a causal relation. ${ }^{8}$ The correlation coefficient is $>0.9$ for the association between tissue glutathione and preoperative ejection fraction, but the data plot (fig 1 in De Vecchi et al's paper) demonstrates that they are highly correlated because of polarised grouping of results at low and high ejection fractions, rather than a continuous linear spread. Finally, the clinical outcome in these patients undergoing first time elective revascularisation must be considered. The mortality in the first postoperative month was $10 \%$; more than $50 \%$ of patients required postoperative inotropic support, and more than $20 \%$ needed balloon counterpulsation. However, the authors state that "no patient had electrocardiographic changes and enzyme elevation suggestive of a perioperative myocardial infarction". The above notwithstanding, and despite using the Buckberg regimen, the myocardial protection achieved in the patients with lower ejection fractions in this series is questionable.

Despite some weaknesses, and assuming the data are repeatable, De Vecchi and colleagues have highlighted an important fact-patients with poor ventricular function have lower antioxidant defences and are less well able to counteract the oxidant stresses of ischaemia and reperfusion.

Specialist Registrar in Cardiothoracic Surgery,

D P JENKINS

Harefield Hospital, Harefield, Middlesex UB9 6SH, UK

1 De Vecchi E, Pala MG, Di Credico G, et al. Relation between left ventricular function and oxidative stress in patients undergoing bypass surgery. Heart 1998;79:242-7.

2 Menasche P, Piwnica A. Free radicals and myocardial protection: a surgical viewpoint. Ann Thorac Surg 1989;47:939-45.

3 Braunwald E, Kloner RA. Myocardial reperfusion: a double edged sword. $\mathcal{F}$ Clin Invest 1985;76:1713-19.

4 Bolli R. Mechanisms of myocardial “stunning”. Circulation 1990;82:723-38. 5 Royston D, Fleming JS, Desai JB, et al. Increased production of peroxidation products associated with cardiac operations. Evidence for free radical generation. F Thorac Cardiovasc Surg 1986;91:759-66.

6 Ferrari R, Alfieri O, Curello S, et al. Occurrence of oxidative stress during reperfusion of the human heart. Circulation 1990;81:201-11.

7 reperfusion of the human heart. Circulation $1990 ; 81: 201-11$. allopurinol in coronary artery surgery. Br Heart $\mathcal{f} 1994 ; 71: 177-81$.

allopurinol in coronary artery surgery. Br Heart $\mathcal{f} 1994 ; 71: 177-81$.
Altman DG. Correlation and regression. In: Practical statistics for medical Altman DG. Correlation and regression. In:
research. London: Chapman and Hall, 1991. 